\title{
Relación de la menarquia precoz con disminución de la reserva ovárica en la vida adulta. Revisión sistemática y meta-análisis
}

\author{
Juan Enrique Schwarze M. MSCE ${ }^{1,2}$, Esteban Alwane $0^{3}{ }^{3}$, Sonia Villa $V^{2}$, Carolina \\ Ortega H. ${ }^{2}$ Ricardo Pommer T.2 \\ ${ }^{1}$ Departamento Clínico de Obstetricia y Ginecología, Universidad de Santiago. ${ }^{2}$ Unidad Medicina Reproductiva Clínica \\ Monteblanco. ${ }^{3}$ Programa de Formación de Especialistas Universidad de Santiago.
}

\section{RESUMEN}

Antecedentes: La identificación de factores que pudieran afectar la reserva ovárica permitiría predecir el resultado a la hiperestimulación ovárica en las técnicas de reproducción asistida, y en forma independiente a la edad, el éxito de estos procedimientos. Se ha propuesto que la presencia de menarquia precoz, definido como su aparición antes de los 12 años, sería uno de los factores predictivos de baja reserva ovárica. Objetivo: Determinar si existe asociación entre menarquia precoz y disminución de la reserva ovárica futura. Método: Se realizó una revisión sistemática de la literatura en las bases de datos MEDLINE y EMBASE entre los años 2000 y 2013 con los conceptos "premature menarchae" y "ovarian reserve". Sólo 3 artículos cumplieron los criterios de selección, con un total de 2.470 pacientes. Resultado: El meta-análisis mostró una asociación significativa entre menarquia temprana y disminución de la reserva ovárica en la vida adulta (OR 1,89; IC95\% 1,52-2,35; p=0,001). Conclusión: De confirmarse la relación entre disminución de reserva ovárica en pacientes que presentaron menarquia precoz, será fundamental que ésta información sea debidamente registrada y evaluada dentro de la consejería preconcepcional.

\section{PALABRAS CLAVE: Reserva ovárica, menarquia temprana, revisión sistemática}

\section{SUMMARY}

Background: The identification of predictors of controlled ovarian hyperstimulation could predict the outcome of assisted reproductive technologies procedures, and independently of age, predict the success of said procedures. It has been suggested that premature menarchae, i.e. below 12 years of age, predicts diminished ovarian reserve. Objective: To determine if there is an association between premature menarche and diminished ovarian reserve. Methods: We performed a systematic review in MEDLINE and EMBASE, for articles published between 2000 and 2013, with key words "premature menarchae" and "ovarian reserve". Three articles fulfilled the inclusion criteria, including a total of 2,470 patients. Results: The meta-analysis of the results demonstrated an association between premature menarchae and diminished ovarian reserve (OR 1.89; $95 \% \mathrm{Cl} 1.52-2.35 ; \mathrm{p}=0.001$ ). Conclusion: If our findings are confirmed, it would be fundamental to include this information in any routine gynecological visit.

KEY WORD: Ovarian reserve, premature menarchae, systemic review 


\section{INTRODUCCIÓN}

Entendemos como reserva ovárica a la suma de folículos primarios y primordiales presentes en los ovarios $(1,2)$. Su principal importancia clínica es predecir la respuesta ovárica a la hiperestimulación ovárica controlada, y así, en forma indirecta, predecir la posibilidad de embarazo de un procedimiento de reproducción asistida $(3,4)$. Además, el diagnóstico de reserva ovárica disminuida precede en aparentemente diez años la aparición de menopausia (5).

Existen varios exámenes para diagnosticar una disminución de la reserva ovárica, entre los más usados están: determinación de niveles plasmáticos de FSH y estradiol, hormona anti-mülleriana y el recuento de folículos antrales (5).

Los factores de riesgo descritos para disminución de reserva ovárica son, entre otros, el tabaquismo y factores genéticos intrínsecos (6). Dentro de estos factores genéticos se ha descrito la presencia de menarquia precoz.

El objetivo de esta revisión sistemática es responder la pregunta si las pacientes que tuvieron una menarquia precoz tienen un mayor riesgo de presentar una disminución en su reserva ovárica en la vida adulta.

\section{MATERIALES Y MÉTODOS}

Se realizó una búsqueda en las bases de datos MEDLINE y EMBASE, de artículos de estudios prospectivos o retrospectivos con los siguientes términos en el título o resumen: "menarche"y "ovarian reserve".

Los criterios de inclusión fueron: artículos escritos en inglés o castellano, publicados entre los años 2000 y 2013. Se definió menarquia precoz como la aparición de ésta con menos de 12 años de edad. Se definió baja reserva ovárica como un valor de hormona antimülleriana bajo el p25 de acuerdo a la edad, o mala respuesta a la hipertestimulación ovárica controlada, de acuerdo con los criterios de Boloña (7). Se definió falla ovárica prematura como el cese de la menstruación por un año en un paciente con menos de 40 años. Se excluyeron estudios secundarios (meta-análisis, revisiones sistemáticas) y estudios sin grupo control. También revisamos la lista de la bibliografía de los artículos incluido, en búsqueda de otros artículos pertinentes y no identificados previamente.

Una vez seleccionados los artículos por el título y resumen, éstos fueron revisados por dos de los autores (EA, JES) para determinar su inclusión en el estudio. En el caso de divergencia de opiniones, un tercer autor (SV) decidió finalmente su inclusión o exclusión.

Los datos extraídos fueron traspasados a una forma estándar, que incluía referencia, objetivo, población, determinación de menarquia precoz, deter- minación de reserva ovárica, frecuencia de reserva ovárica alterada y comentarios.

El meta-análisis fue realizado con Stata (Statacorp, EEUU). La heterogeneicidad entre estudios fue evaluada mediante prueba de Chi cuadrado. Un modelo de efecto fijo fue usado para el meta-análisis y, utilizando la prueba de Mantel-Haensze se obtuvo una Razón de Odds (OR), con un intervalo de confianza del 95\% (IC95\%). Los resultados se expresaron gráficamente como figura de bosque.

\section{RESULTADOS}

La primera búsqueda arrojó 15 títulos de artículos elegibles. Luego de revisar los artículos en extenso por dos de los autores, sólo tres cumplieron los criterios de inclusión (Figura 1 y Tabla I). La suma de las pacientes evaluadas fue de 2450. A continuación presentamos los principales resultados de los 3 trabajos seleccionados.

Sadrzadeh y cols (8), realizaron un estudio de caso control, ocupando una muestra de la cohorte de 26.428 mujeres incluidas en el proyecto OME$\mathrm{GA}$, de técnicas de reproducción asistida. Ellos evaluaron a pacientes ya sea con síndrome de ovarios poliquísiticos (controles) y pacientes con reserva ovárica disminuía (casos), y que recibieron ovocitos donados, o respondieron con menos que tres ovocitos a la hiperestimulación ovárica controlada, en tres categorías de edad de menarquia: $\leq 11$ años, $12-14$ años, y $\geq 15$ años. De las 124 mujeres con menarquia $\leq 11$ años, $83(67 \%)$ tenia reserva ovárica disminuida, comparada con 41 (37\%) de las 123 mujeres con menarquia $\geq 14$ años.

Chang y cols (9), realizaron un estudio retrospectivo de cohorte, utilizando la población de la cohorte de cáncer de Corea, entre el año 2002 y 2003. Se definió como disminución de la reserva ovárica a la falla ovárica prematura, o menopausia antes de los 44 años. Ocuparon como definición de menarquia temprana la aparición de la primera menstruación antes de los 13 años de edad. De las 2.668 pacientes de la cohorte inicial, 1.281 fueron incluidas en el análisis. De las 168 pacientes con menarquia $\leq 13$ años, 81 (50\%) presentaron menopausia antes de los 44 años, mientras que de las 1.533 mujeres con menarquia $\geq 14$ años, 585 (38\%) presentaron menopausia antes de los 44 años.

Weghofer y cols (10), realizaron un estudio retrospectivo de cohorte, con un total de 502 pacientes. Definieron menarquia temprana a la aparición de la primera regla antes de los 13 años. La disminución de la reserva ovárica se determinó con un nivel de hormona antimülleriana menor al percentil de 25 de la categoría etaria de la mujer. De las 193 mujeres con menarquia precoz, 121 (63\%) presentaron reserva ovárica disminuida; mientras que de las 309 mujeres con menarquia $\geq 14$ años, $158(51 \%)$ presentaron reserva ovárica disminuida. 
16 artículos potencialmente relevantes al buscar en PubMed y Embase.

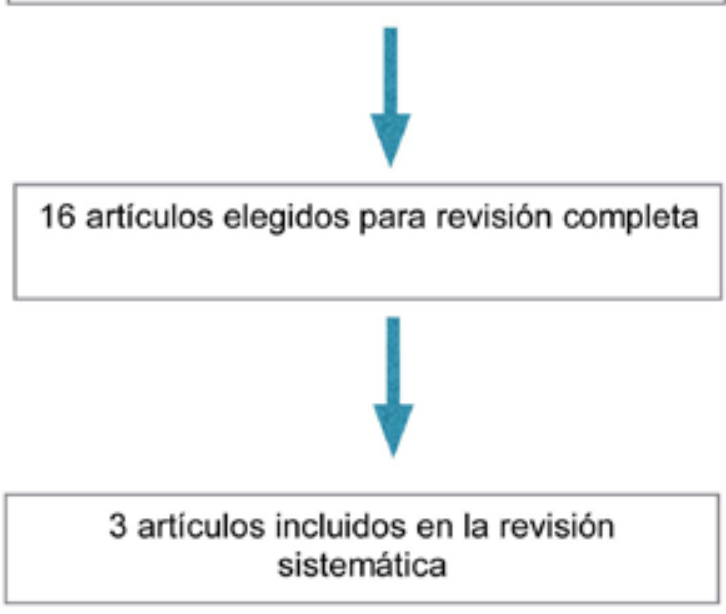

13 artículos excluidos:

12 artículos correspondian a estudio en pacientes con cáncer

1 artículo no presenta datos completos

Figura 1. Flujograma de revisión sistemática.

Tabla I

RESUMEN DE LOS TRABAJOS SELECCIONADOS

\begin{tabular}{lll}
\hline Autor & Metodología & Hallazgos \\
\hline Sadrzadehy cols (8) & Estudio de caso y control & $83(67 \%)$ mujeres con reserva ovárica \\
& & disminuida (ovorreceptoras o respuesta de \\
& $\leq 3$ ovocitos) tuvieron menarquia $\leq 11$ años, \\
& mientras que $54(44 \%)$ mujeres con SOP \\
tuvieron menarquia a los $\leq 11$ años
\end{tabular}

Chang y cols (9) Estudio transversal de mujeres 81 (48\%) mujeres con menopausia $\geq 45$ años pertenecientes a la Cohorte Multicéntrica tuvieron menarquia $\geq 13$ años; comparado con de Cáncer de mujeres Coreanas 585 (38\%) mujeres con menarquia $\geq 14$ años

Weghofery cols (10) Estudio de cohorte retrospectivo, en el que se evaluó consecutivamente a 502 parejas infértiles

$121(62,7 \%)$ de mujeres con menarquia precoz (<11 años) tuvieron reserva ovárica disminuida (nivel de AMH bajo el p25 para la edad), comparada con $158(51,1 \%)$ de las mujeres con menarquia después de los 13 años (OR 1,6; IC95\% 1,1-2,3)

Individualmente, los artículos encontraron diferencia estadísticamente significativa en el riesgo de las pacientes con menarquia precoz de presentar reserva ovárica disminuida. Al realizar el meta-análisis, encontramos que las pacientes con menarquia precoz tenían un riesgo aumentada de tener una disminución de la reserva ovárica en su vida adulta. El grupo con menarquia precoz estaba constituido por 485 pacientes con un OR para reserva ovárica disminuida de 1,89 (IC95\% 1,522,$35 ; p=0,001$ ) (Figura 2). 


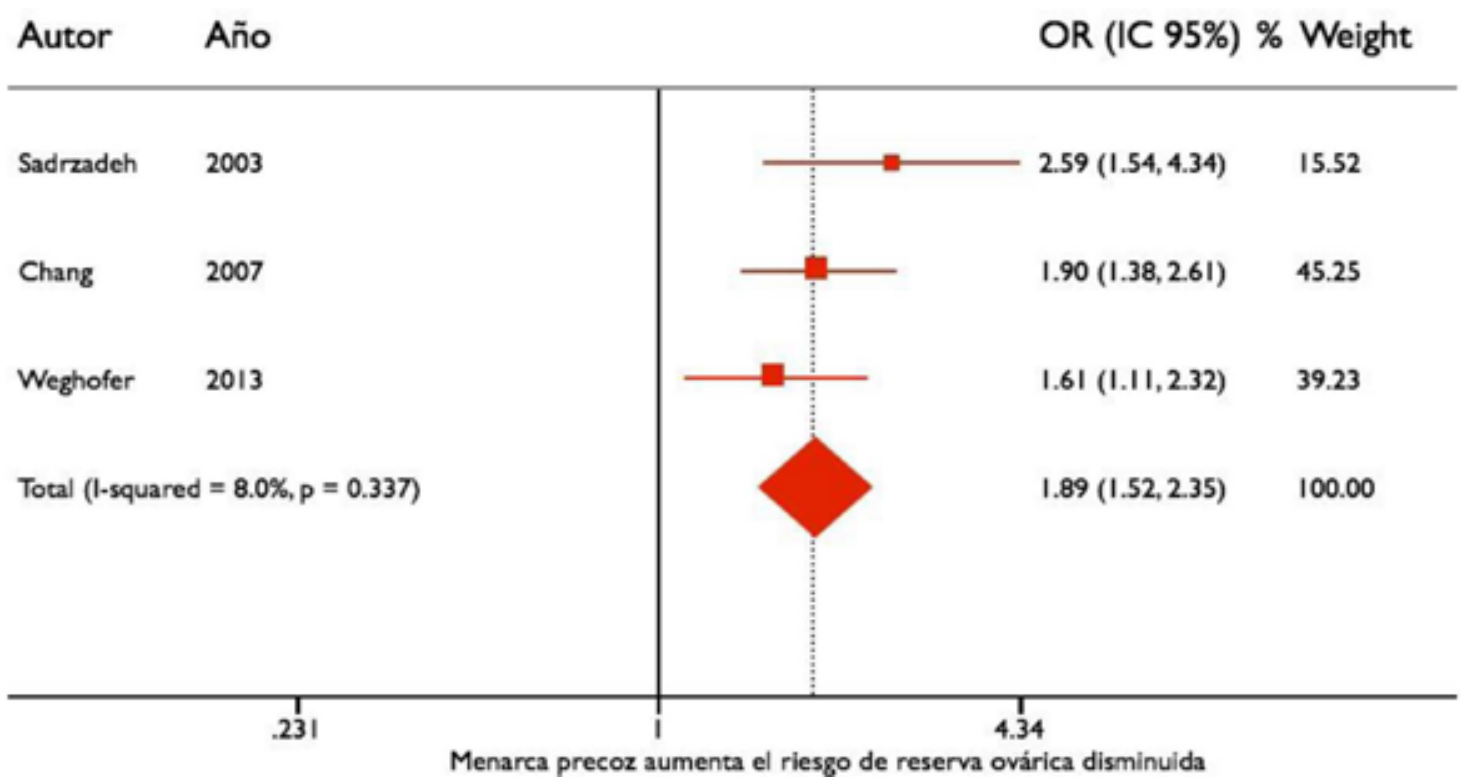

Figura 2. Gráfico de bosque de OR de reserva ovárica disminuida en mujeres con menarquia precoz.

\section{DISCUSIÓN}

El resultado de nuestro meta-análisis demuestra que existe una asociación estadísticamente significativa entre la menarquia temprana y la disminución prematura de la reserva ovárica en la vida adulta. Esta relación se podría explicar, por un factor genético del desarrollo ovárico, que determinaría una vida reproductiva finita, con un pool ovocitario ya definido desde la vida intrauterina, para cada mujer. Por lo tanto, al presentar una menarquia de forma temprana, su capacidad reproductiva se adelantaría, como un bloque, produciendo una menor reserva ovárica en etapas más tempranas que en la población general. Esto es de suma importancia, debido a que las pacientes que presenten esta condición, podrían tener una fecundidad disminuida durante la vida adulta. Por lo tanto, al determinar la menarquia temprana como un factor de riesgo que podría influir en el éxito reproductivo, tanto en embarazos espontáneos como asistidos, los médicos a cargo de estas pacientes, pediatras y/o ginecólogos, deberían dar una consejería preconcepcional, y orientarlas a no postergar su maternidad, y/o criopreservar ovocitos.

Una ventaja de esta revisión es la homogeneidad en los resultados de los distintos estudios, realizados con diferentes grupos tanto étnicos como de casos, e incluso con distintas determinaciones de menarquia precoz. Probablemente la principal desventaja es la heterogeneidad en la definición o estimación de baja reserva ovárica, ya que algunos casos se basaron en el recuerdo de la paciente de la edad de su menopausia, y en otros casos fueron condiciones más objetivas como el número de ovocitos recuperados luego de una hiperstimulación ovárica controlada.

Producto de cambios demográficos y ambientales poco claros, se ha generado una transición en las niñas alrededor del mundo, en donde la menarquia y la pubertad son de aparición mas temprana que en épocas anteriores (11). Estos cambios poblacionales podrían, en un futuro, determinar un grupo mayor de mujeres con una reserva ovárica disminuida en etapas más tempranas de la vida. Esto aumentaría las necesidades de técnicas de reproducción asistida de alta complejidad, que no podrían se abarcadas por el sistema de salud actual, encareciendo aún más los costos de los tratamientos y requiriendo un mayor número de profesionales a cargo del tema.

\section{CONCLUSIÓN}

De confirmarse la relación entre disminución de reserva ovárica en pacientes que presentaron 
menarquia precoz, será fundamental que ésta información sea debidamente registrada y evaluada dentro de la consejería preconcepcional.

\section{REFERENCIAS}

1. Corson SL. Screening for ovarian reserve. Fertil Steril 1995;64(1):222-3.

2. Scott RTJr, Hofmann GE. Prognostic assessment of ovarian reserve. Fertil Steril 1995;63(1):1-11.

3. Scott RT, Opsahl MS, Leonardi MR, Neall GS, Illions EH, Navot D. Life table analysis of pregnancy rates in a general infertility population relative to ovarian reserve and patient age. Hum Reprod 1995;10(7):1706-10.

4. Bancsi LF, Broekmans FJ, Eijkemans MJ, de Jong FH, Habbema JD, te Velde ER. Predictors of poor ovarian response in in vitro fertilization: a prospective study comparing basal markers of ovarian reserve. Fertil Steril 2002;77(2):328-36.

5. Broekmans FJ, Kwee J, Hendriks DJ, Mol BW, Lambalk CB. A systematic review of tests predicting ovarian reserve and IVF outcome. Hum Reprod Update 2006;12(6):685-718.

6. Sharara Fl, Beatse SN, Leonardi MR, Navot D, Scott RT. Cigarette smoking accelerates the development of diminished ovarian reserve as evidenced by the clomiphene citrate challenge test. Fertil Steril 1994;62(2):257-62.

7. Ferraretti AP, La Marca A, Fauser BC, Tarlatzis B, Nargund G, Gianaroli L, ESHRE working group on Poor Ovarian Response Definition. ESHRE consensus on the definition of 'poor response' to ovarian stimulation for in vitro fertilization: the Bologna criteria. Hum Reprod 2011;26(7):1616-24.

8. Sadrzadeh S, Klip WA, Broekmans FJ, Schats R, Willemsen WN, Burger CW, Van Leeuwen FE, Lambalk CB, OMEGA Project group. Birth weight and age at menarche in patients with polycystic ovary syndrome or diminished ovarian reserve, in a retrospective cohort. Hum Reprod 2003;18(10):2225-30.

9. Chang SH, Kim CS, Lee KS, Kim H, Yim SV, Lim YJ, Park SK. Premenopausal factors influencing premature ovarian failure and early menopause. Maturitas 2007;58(1):19-30.

10. Weghofer A, Kim A, Barad DH, Gleicher N. Age at menarche: a predictor of diminished ovarian function? Fertil Steril 2013;100(4):1039-43.

11. McDowell MA, Brody DJ, and Hughes JP. Has age at menarche changed? Results from the National Health and Nutrition Examination Survey (NHANES) 1999-2004. J Adolesc Health 2007;40(3):227-31. 